\title{
Towards a new measurement of the neutron electric dipole moment
}

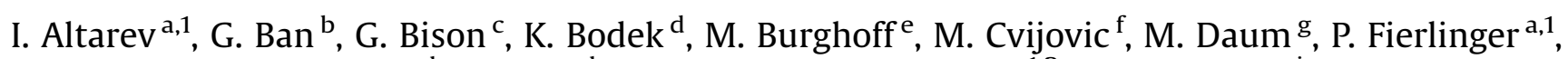 \\ E. Gutsmiedl ${ }^{\text {a }}$, G. Hampel ${ }^{\text {h }}$, W. Heil ${ }^{\text {h }}$, R. Henneck ${ }^{g}$, M. Horras ${ }^{\text {a, } 1,2}$, N. Khomutov ${ }^{\mathrm{i}}$, K. Kirch ${ }^{\text {g, }}$, \\ St. Kistryn ${ }^{\text {d }}$, S. Knappe-Grüneberg ${ }^{\text {, }}$, A. Knecht ${ }^{\text {g,3 }}{ }^{\text {, P. Knowles }}{ }^{\text {, }}$, A. Kozela d,4 J.V. Kratz ${ }^{\text {h }}$, F. Kuchler ${ }^{\text {a,1 }}$, \\ M. Kuźniak ${ }^{\mathrm{d}, 2}$, T. Lauer ${ }^{\mathrm{h}}$, B. Lauss ${ }^{\mathrm{g}}$, T. Lefort ${ }^{\mathrm{b}}$, A. Mtchedlishvili ${ }^{\mathrm{g}}$, O. Naviliat-Cuncic ${ }^{\mathrm{b}}$, S. Paul ${ }^{\mathrm{a}, 1}$,
} A.S. Pazgalev ${ }^{\mathrm{f}}$, G. Petzoldt ${ }^{\mathrm{g}}$, E. Pierre ${ }^{\mathrm{b}, 2}$, C. Plonka-Spehr ${ }^{\mathrm{j}, 5}$, G. Quéméner $^{\mathrm{k}}$, D. Rebreyend $^{\mathrm{k}}$, S. Roccia $^{\mathrm{k}}$, G. Rogel ${ }^{\mathrm{b}}$, T. Sander-Thoemmes ${ }^{\mathrm{e}}$, A. Schnabel ${ }^{\mathrm{e}}$, N. Severijns ${ }^{1}$, Yu. Sobolev ${ }^{\text {h,6 }}$, R. Stoepler ${ }^{\mathrm{a}, 1}{ }^{1}$ L. Trahms $^{\mathrm{e}}$, A. Weis ${ }^{\mathrm{f}}, \mathrm{N}$. Wiehl ${ }^{\mathrm{h}}$, J. Zejma ${ }^{\mathrm{d}}, \mathrm{G}$. Zsigmond ${ }^{\mathrm{g}}$

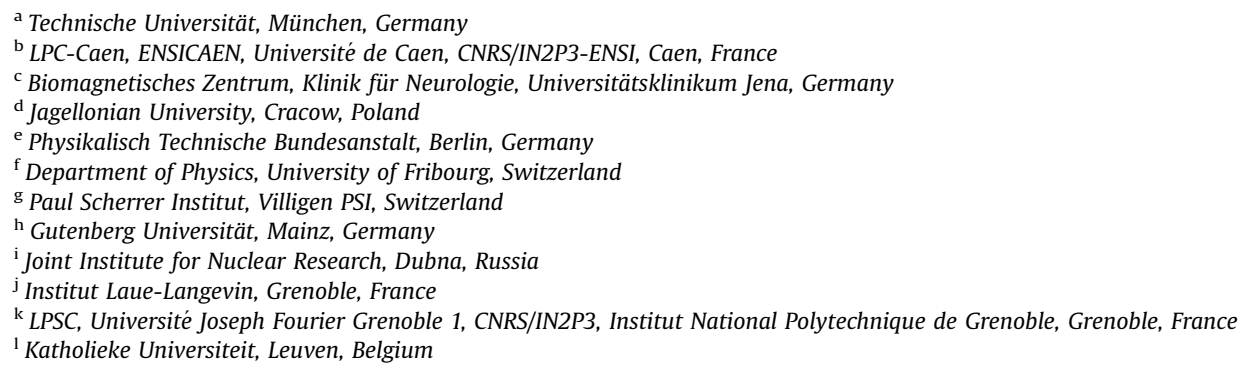

\section{Introduction}

As it would for any particle, an electric dipole moment of the neutron (nEDM) violates both parity $(\mathscr{P})$ and time reversal $(\mathscr{T})$ symmetry $[1,2]$, and, via the $\mathscr{C} \mathscr{P} \mathscr{T}$-theorem, $\mathscr{C} \mathscr{P}$. Assuming

\footnotetext{
* Corresponding author.

E-mail address: klaus.kirch@psi.ch (K. Kirch).

${ }^{1}$ Also Excellence-Cluster Exc153 'Origin and Structure of the Universe', Garching.

${ }^{2}$ Also at Paul Scherrer Institut.

${ }^{3}$ Also at University of Zürich.

${ }^{4}$ At Institute for Nuclear Physics, Cracow.

${ }^{5}$ Now at Gutenberg Universität, Mainz.

${ }^{6}$ Also at PNPI Gatchina.
}

$\mathscr{C} \mathscr{P} \mathscr{T}$-invariance and an initially matter-antimatter symmetric universe, additional $\mathscr{C} \mathscr{P}$-violation (beyond that established in the electro-weak sector of the particle physics Standard Model) is necessary to explain the observed baryon asymmetry [3]. The nEDM is one of the most sensitive and promising probes for such additional $\mathscr{C} \mathscr{P}$-violation. The most recent experimental limit, $\left|d_{n}\right|<2.9 \times 10^{-26}$ e cm [4], continues to challenge our understanding of fundamental physics, in particular via the so-called "Strong $\mathscr{C} \mathscr{P}$-Problem" and the "SUSY $\mathscr{C} \mathscr{P}$-Problem". For reviews, see Refs. [5-7].

Today, various efforts are underway to improve the sensitivity of the experimental nEDM search. A necessary step in all approaches is the increase of neutron statistics and thus improved UCN production schemes are required. Our nEDM collaboration is heavily engaged with the development and commissioning of new 
solid deuterium based UCN sources [8,9]. Distinctive features of our nEDM approach are the use of UCN in vacuum in an apparatus at ambient temperature, the continued use of Ramsey's separated oscillatory field method, and the addition of multiple complementary magnetometry methods.

Our project is pursued in three phases:

- Phase I (at ILL, in progress until the end of 2008): Research and development on the improvements of the RAL/Sussex/ILL apparatus $[4,10]$.

- Phase II (at PSI, 2009-2011): Measuring with an upgraded RAL/ Sussex/ILL apparatus at the PSI UCN source aiming at a sensitivity of $5 \times 10^{-27} \mathrm{e} \mathrm{cm}$.

- Phase III (2011-2015): Measurement with a new apparatus aiming at $5 \times 10^{-28}$ e cm sensitivity.

\section{Phase I: ongoing R\&D}

We are concentrating on tests and improvements of the RAL/ Sussex/ILL apparatus [4,10] at ILL Grenoble. Major R\&D topics are the development of new magnetometry options; improved magnetic field control; new UCN detection techniques; new suitable materials for insulators, electrodes, shutters, guides, coatings; new data acquisition and monitoring systems; and improved calculational and simulational tools.

Phase II foresees the addition to the system of an array of laserpumped optical Cs magnetometers (CsM). The CsM array, located in proximity to still outside the UCN storage chamber, will yield a time-resolved magnetic field map of the immediate surroundings of the measurement volume. It can be used for magnetic field tracking in order to apply phase corrections to the neutron Ramsey pulses, and in a slow feedback system to stabilize the magnetic field and first order gradients. The effect of feedback stabilization of the main magnetic field using a CsM prototype system is displayed in Fig. 1. Two time series are shown: each series shows the $\mathrm{Hg}$ comagnetometer average precession frequency for the magnetic field within the neutron confinement volume as determined by the existing $\mathrm{Hg}$ magnetometry system. The apparatus was run without neutrons, but using all steps of the measurement process, so that a $180 \mathrm{~s}$ free precession time for field averaging was embedded within a $223 \mathrm{~s}$ cycle time. The two time series above each represent a little more than $8 \mathrm{~h}$ of data. The top trace shows the field fluctuations measured by the comagnetometer when the system was running without intervention from the Cs magnetometry. It has an Allan

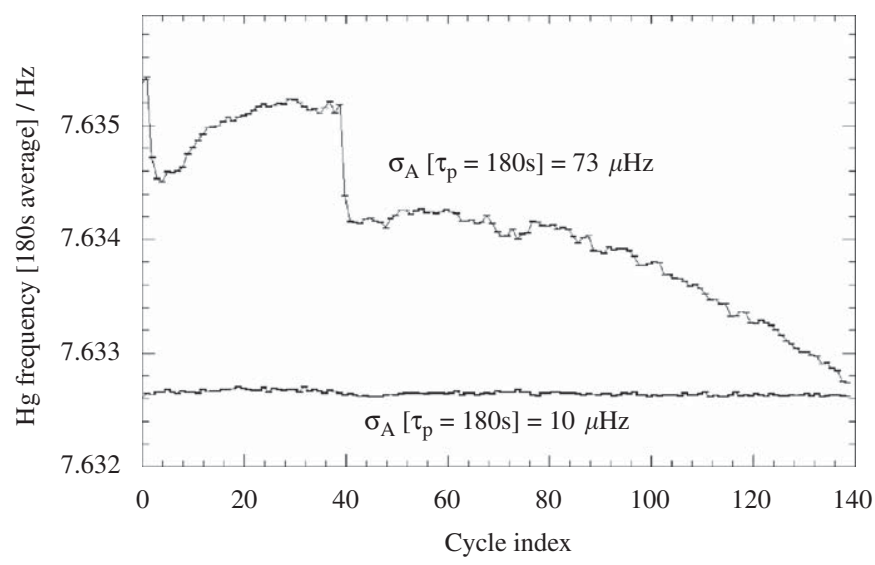

Fig. 1. A time series (about $8 \mathrm{~h}$ ) for the $\mathrm{Hg}$ comagnetometer measured precession frequency for unstabilized (top trace) and Cs-feedback stabilized (bottom trace) conditions within the EDM apparatus. An improvement factor of about 7 is indicated by the Allan standard deviations calculated for 180 s samples. variance of $73 \mu \mathrm{Hz}$, and a precision per point of between 2 and $5 \mu \mathrm{Hz}$ (the nominal field is $1 \mu \mathrm{T}$, so $2 \mu \mathrm{Hz}$ represents about $270 \mathrm{fT}$ ). The bottom trace shows the gain in system stability when the Cs magnetometry was used in feedback loops improvised into the system with the aim of stabilizing the field. The difference in field stability is well represented by the Allan variance of $10 \mu \mathrm{Hz}$, a factor of 7 improved from the free field variations. The stabilization procedure was made only on the field magnitude, and not on the field components, and the procedure was not optimized. While the free running ${ }^{199} \mathrm{Hg}$ comagnetometer is affected by jumps in the external magnetic field, the Cs feedback effectively suppresses these jumps. For more details concerning Cs magnetometry, see [11]. R\&D on improving the sensitivity of the ${ }^{199} \mathrm{Hg}$ comagnetometer [12] is also ongoing [13], as well as studies preparing the use of large area ${ }^{3} \mathrm{He}$ magnetometers in Phase III, based on Ref. [14].

Considerable progress has been made in the development of efficient UCN detectors capable of high rate operation, and UCN polarimetry [15]. A report on ${ }^{6} \mathrm{Li}$ doped glass scintillators is found in Ref. [16]. Effort is also devoted to the development of a velocity sensitive UCN detector [17]. The measurement of the nEDM as a function of UCN velocity could be a powerful systematic check of any given result.

The isolator used as confining wall for UCN in the precession chamber $[4,10]$ is bare quartz (90 neV Fermi potential). Higher Fermi potentials are very desirable since they lead to higher UCN statistics after typical UCN storage times of $150 \mathrm{~s}$. We have developed a technique to coat polystyrene (PS) isolators with deuterated PS (DPS) [18,19]. The performance under high electric fields, with UCN and ${ }^{199} \mathrm{Hg}$ compares favorably with the quartz chamber. For the test of the PS ring we successfully employed UVgrade quartz windows coated by DPE (deuterated polyethylene; using spin-coating to obtain an optically flat surface), since DPS deteriorates under UV illumination, necessary for the comagnetometer. The Fermi potential of DPE was measured to be about $V_{\mathrm{F}}=214 \mathrm{neV}[18,19]$, and that for DPS is $161 \mathrm{neV}[18-20]$.

Fig. 2 displays the result of GEANT4 simulations [21] for detected UCN counts as a function of storage time. The lowest curve corresponds to the measured performance at ILL, the next higher for the quartz chamber at PSI which again improves when going to

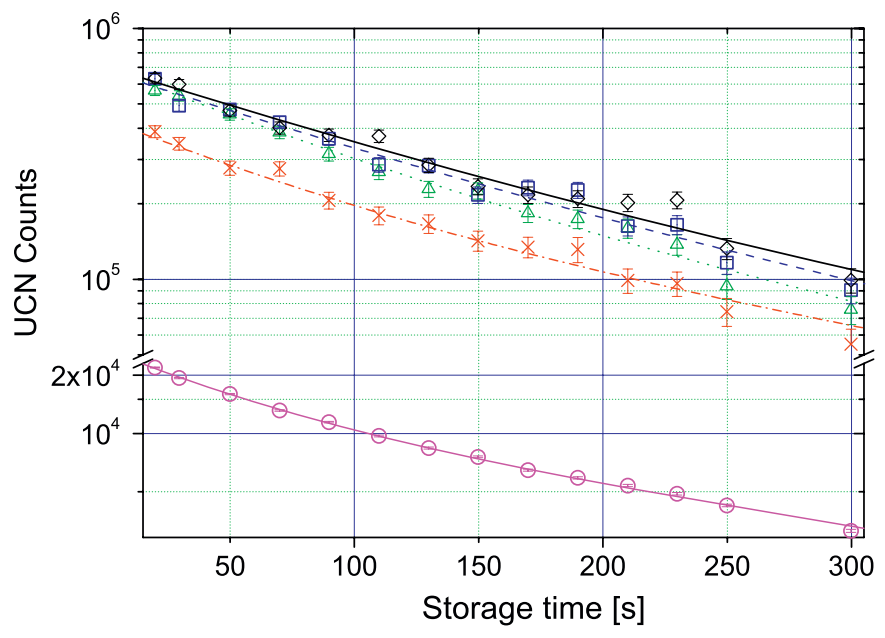

Fig. 2. Simulation (using Ref. [21]) of neutron storage behaviour in the existing nEDM apparatus, placed $1 \mathrm{~m}$ above the PSI UCN beamline: quartz insulator + diamond-like carbon (DLC) coated electrodes (red $\times$, dash-dotted line), DPScoated insulator + DLC-coated electrodes (green $\triangle$, dotted line), diamond coated insulator + DLC electrodes (blue $\square$, dashed line), diamond coated insulator and electrodes (black $\diamond$, solid line). Experimental curve from ILL shown for comparison (magenta $\circ$, solid line) [18]. (For interpretation of the references to colour in this figure legend, the reader is referred to the web version of this article.) 
the DPS insulator. Further improvement from higher Fermi potential materials is possible but would yield only small additional gain. The simulational model has been calibrated with the measured storage, filling, and emptying time values of the ILL EDM experiment and has the material properties as parameters. Typically the Fermi potentials of the used materials are sufficiently well known whereas the loss parameters (here $\eta \sim 1-4 \times 10^{-4}$ ) and the degrees of diffuse reflection (1-10\%) were adapted to fit the data. More details of the simulation are found in Ref. [18]. Experimental confirmation of the simulated gain factors comes from the observed $50 \%$ increase in UCN counts after $150 \mathrm{~s}$ storage time at ILL when replacing the quartz ring by the PS ring.

\section{Phase II: measurement at PSI}

The concept for the Phase II setup is shown in Fig. 3. From left to right, the UCN pass through the superconducting (SC) polarizer magnet, a custom designed UCN vacuum valve, a UCN switch valve with its connections up into the UCN trap, down into the detection system and horizontally through-going to a second UCN vacuum valve. The maximum number of UCN in the experiment is obtained by minimizing the vertical distance between the storage chamber and the horizontal input guide. Not shown here are the vacuum system, thermal housing, and external field compensation coil system.

The SC magnet is used to provide fully polarized UCN and also to minimize losses in the unavoidable safety window of the UCN source, which is placed in the region of maximal magnetic field. Ultracold neutrons passing the SC magnet have highest velocities in the maximal field region and can therefore more easily traverse the material, a concept first described in Ref. [22]. The expected UCN performance for the SC magnet at the PSI UCN source has been carefully simulated.

The UCN vacuum valves have been derived from standard DN200 components ${ }^{7}$ but include efforts to minimize slits and to coat all UCN contacting surfaces with vacuum-arc deposited diamond-like carbon (see Ref. [23] and references therein). In their closed (vacuum tight) position these valves are expected to be suitable for UCN storage; in their open position, two specially designed rings spread over the gap between the connected UCN guides (ID $180 \mathrm{~mm}$ ) and minimize slit widths to about $0.1-0.2 \mathrm{~mm}$.

The UCN switch valve is used to direct the UCN from the polarizer to the experiment either up towards the EDM chamber or straight to a second (test) beam position. Besides filling the EDM experiment, the switch can be moved to an emptying position which connects the vertical guide from the EDM chamber to the detection system below. Another switch feature is dedicated to UCN intensity monitoring. Monitoring is important, since there is not yet experience with the UCN production stability from the pulsed spallation UCN source [8]. The switch will provide a well-defined connection between the horizontal guide following the polarizer and the detection system during the filling the EDM chamber. Because the horizontal guides have $180 \mathrm{~mm}$ inner diameter and the vertical guide to the EDM chamber only $\sim 70 \mathrm{~mm}$, there is sufficient space for a channel of $\sim \mathrm{cm}^{2}$ crosssection. This small channel does not considerably affect the UCN density in the EDM experiment, but does allow $10^{6}-10^{7}$ UCN to accumulate in the detection system over the $\sim 40$ s filling time of the experiment. When the EDM chamber is filled, the guide between the first UCN vacuum valve and the switch holds of order $10^{7}$ UCN. These UCN must be treated with care since any leakage around the switch into the detection system permits them to, at

${ }^{7}$ VAT Series 17, www.vatvalve.com.

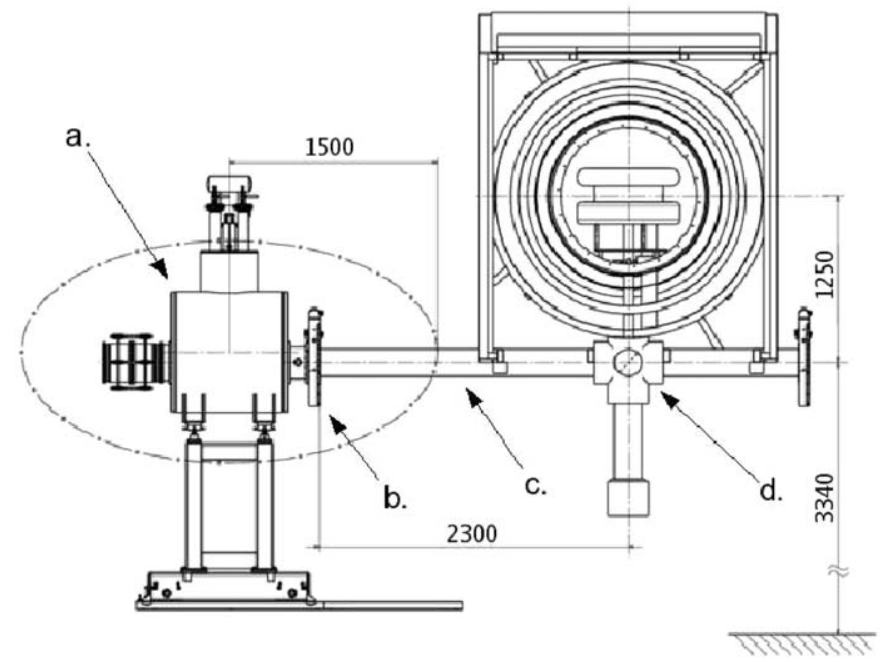

Fig. 3. A schematic of the former RAL/Sussex/ILL apparatus moved to PSI and installed with SC polarizer magnet (a), VAT valve (b), UCN guide system (c), UCN switch (d): spectrometer (up), detection system (down) and test beam port (straight). The ellipse around the magnet indicates the $1 \mathrm{G}$ line.

worse, falsify the later EDM neutron counting, and at best increase the background; thus, they have to disappear within a short time, order $\sim 10$ s following filling. However, they should not simply be dumped, since detecting them will provide another UCN monitor for the EDM experiment. It is presently under discussion whether the detection system below the switch will be used to detect these "guide neutrons" or if another detector further downstream of the switch will be used. Obviously, the high monitor count rates are quite demanding for the detection system (they are 1-2 orders of magnitude larger than the count rates of stored UCN from the EDM chamber, see Fig. 2) but can be handled [16].

A sensitivity increase by a factor of 5 is anticipated due to the higher UCN intensity (cf. Fig. 2 for the factor of about 30 in UCN counts between the quartz chamber at ILL and the DPS chamber at PSI) and better systematic control (based on the results of present $\mathrm{R} \& \mathrm{D}$, mainly magnetometry and field stabilization).

\section{Phase III: double chamber setup}

The next generation spectrometer will have a vertical field configuration and cylindrical, vertically stacked double UCN chambers inside a horizontal, cylindrical, multilayer $\mu$-metal shield. A conceptual layout of the new nEDM apparatus is shown in Fig. 4. The additional improvement in sensitivity is mainly expected due to a larger trap volume $(x \sqrt{3})$, better adaptation to the UCN source $(x \sqrt{3})$, longer running time $(x \sqrt{3})$, and higher electric field $(x 2)$.

Better control over systematics will be secured by a double UCN chamber geometry (the double chamber concept was first realized for a neutron EDM search in Ref. [24]), by improved control of the magnetic field and gradients (aided by a new magnetic shielding), a field stabilization system, and an enhanced magnetometry system. An additional feature under investigation is a velocity sensitive UCN detection which would allow studying correlations between EDM signals and UCN velocity. This might be particularly interesting because various systematic effects depend on UCN velocity.

It is planned to combine comagnetometry (improved ${ }^{199} \mathrm{Hg}$ magnetometry, potentially ${ }^{129} \mathrm{Xe}$ and ${ }^{3} \mathrm{He}$ ), with field control by multiple Cs-sensors [11] and two large volume ${ }^{3} \mathrm{He}$ magnetometers [14], also read by Cs magnetometers. 


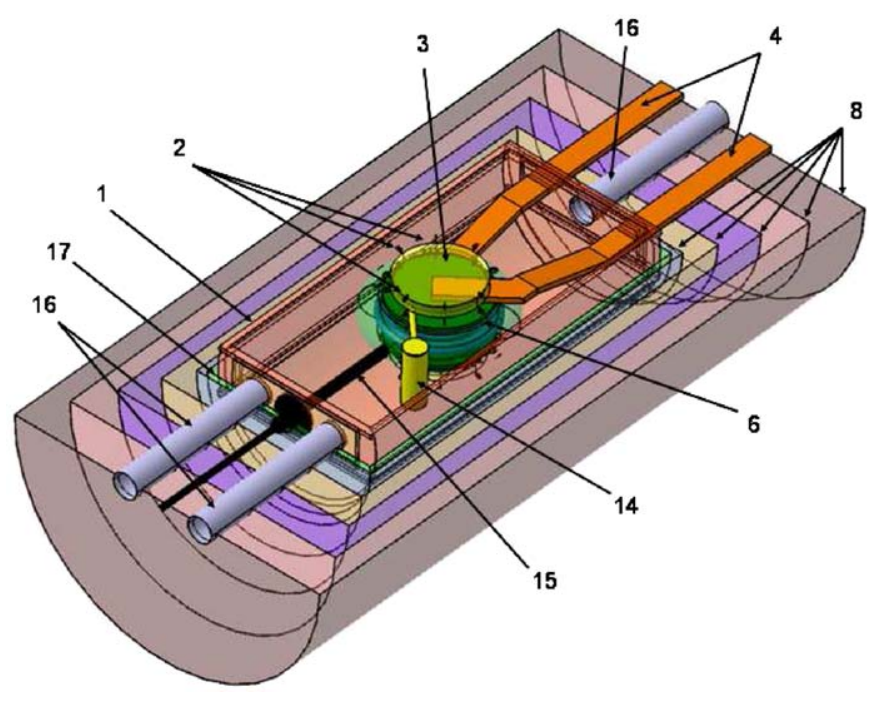

Fig. 4. (Colour online) A view of the n2EDM apparatus from the presently ongoing design study. The numbers label vacuum chamber (1), Cs magnetometer array (2) for both B-field measurement and ${ }^{3} \mathrm{He}$ read-out, the two large ${ }^{3} \mathrm{He}$ magnetometer vessels (3), rectangular shaped UCN guides (4), UCN double-chamber (6), 5-layer $\mu$-metal shield (8), a comagnetometry system (14), HV connection (15), various feedthroughs for pumping and electrical, optical and gas connections (16), and the inner coil system (17).

Our feasibility studies show that the proposed combination of a CsM array and ${ }^{3} \mathrm{He}$ magnetometers is capable of tracing tiny magnetic field variations of $\delta B \approx 2 \mathrm{fT}$ during a typical UCN storage cycle (150-200 s). Field gradients along the main field axis can be monitored with high precision by both magnetometry systems, and other gradients can be detected and tracked by the distributed CsM system. The experiment plans to use two types of Cs sensors, one providing highly accurate scalar information about the B-field magnitude and another designed to measure Bfield vector components.

The ${ }^{199} \mathrm{Hg}$ comagnetometer has so far been used as a very powerful correction device $[4,10]$. With the precision magnetometry around the UCN precession chamber as described above, highly sensitive comagnetometers can then be turned into control devices rather than correction devices. As leading systematic false effects could influence comagnetometers stronger than UCN, e.g., via geometric phases in a conspiracy of electric field and magnetic field gradients [25-28]: the absence of false effects in the comagnetometry might turn out as one of the most powerful proofs for the absence of a false EDM effect in the nEDM measurement.

\section{Conclusion}

We are in the process of improving the in-vacuum technique for the measurement of the neutron electric dipole moment.
The experiment will be performed in steps, delivering first results in about 3-4 years (i.e., 2 years after installation at the running PSI UCN source) with a fivefold improved sensitivity level of $5 \times 10^{-27} \mathrm{ecm}$ and ultimately reaching a sensitivity of $5 \times 10^{-28}$ e cm in $6-8$ years.

In view of the experimental challenges of more precise EDM experiments, the competition of different groups using different, complementary techniques could be crucial. While being on a similar time scale and with comparable sensitivity as other projects [29-31], our approach uses a different technique and offers unique features for systematic control, especially with respect to magnetometry and UCN detection.

\section{Acknowledgements}

We acknowledge the loan of equipment by the RAL/Sussex/ILL collaboration [4], the hospitality and support at PF2 at ILL, as well as the continued support by our institutes and funding agencies. We are especially grateful to the support by technicians, engineers and workshops throughout our collaboration.

\section{References}

[1] L. Landau, Sov. Phys. JETP 5 (1957) 336.

[2] N.F. Ramsey, Phys. Rev. 109 (1958) 225.

[3] A. Sakharov, JETP Lett. 5 (1967) 24.

[4] C.A. Baker, et al., Phys. Rev. Lett. 97 (2006) 131801.

[5] I.B. Khriplovich, S.K. Lamoreaux, CP Violation Without Strangeness, Springer, Berlin, Heidelberg, 1997.

[6] M. Pospelov, A. Ritz, Ann. Physics 318 (2005) 119.

[7] P.G. Harris, arXiv:0709.3100v3 (hep-ex).

[8] A. Anghel, et al., Nucl. Instr. and Meth. A (2009), this issue, doi:10.1016/ j.nima.2009.07.077.

[9] A. Frei, et al., Eur. Phys. J. A 34 (2008) 119.

[10] P.G. Harris, et al., Phys. Rev. Lett. 82 (1999) 904.

[11] P. Knowles, et al., Nucl. Instr. and Meth. A (2009), this issue, doi:10.1016/ j.nima.2009.07.079.

[12] K. Green, et al., Nucl. Instr. and Meth. A 404 (1998) 381.

[13] S. Roccia, Ph.D. Thesis, LPSC, in preparation.

[14] Yu. Borisov, et al., Nucl. Instr. and Meth. A 440 (2000) 483

[15] G. Rogel, Ph.D. Thesis, LPC, in preparation.

[16] G. Ban, et al., Nucl. Instr. and Meth. A (2009), this issue, doi:10.1016/ j.nima.2009.07.083.

[17] A. Knecht, Ph.D. Thesis, Universität Zürich, in preparation.

[18] M. Kuźniak, Ph.D. Thesis, Jagiellonian University, 2008, see ucn.web.psi.ch

[19] K. Bodek, et al., Nucl. Instr. and Meth. A 597 (2008) 222.

[20] S.K. Lamoreaux, ILL Report ILL88LA1T, 1988.

[21] F. Atchison, et al., Nucl. Instr. and Meth. A 552 (2005) 513.

[22] A.P. Serebrov, et al., Nucl. Instr. and Meth. A 545 (2005) 490

[23] F. Atchison, et al., Nucl. Instr. and Meth. A 587 (2008) 82.

[24] I.S. Altarev, et al., Nucl. Phys. A 341 (1980) 269.

[25] J.M. Pendlebury, et al., Phys. Rev. A 70 (2004) 032102.

[26] S.K. Lamoreaux, R. Golub, Phys. Rev. A 71 (2005) 032104.

[27] P.G. Harris, J.M. Pendlebury, Phys. Rev. A 73 (2006) 014101.

[28] A.L. Barabanov, R. Golub, S.K. Lamoreaux, Phys. Rev. A 74 (2006) 052115.

[29] M. van der Grinten, et al., Nucl. Instr. and Meth. A (2009), this issue, doi:10.1016/j.nima.2009.07.040.

[30] 〈http://cns.pnpi.spb.ru/5UCN/articles/Serebrov.pdf 〉.

[31] T.M. Ito, J. Phys. Conf. Ser. 69 (2007) 012037. 